PROCEEDINGS OF THE

AMERICAN MATHEMATICAL SOCIETY

Volume 135, Number 8, August 2007, Pages 2355-2364

S 0002-9939(07)08795-3

Article electronically published on March 22, 2007

\title{
STANLEY-REISNER IDEALS WHOSE POWERS HAVE FINITE LENGTH COHOMOLOGIES
}

\author{
SHIRO GOTO AND YUKIHIDE TAKAYAMA
}

(Communicated by Bernd Ulrich)

\begin{abstract}
We introduce a class of Stanley-Reisner ideals called a generalized complete intersection, which is characterized by the property that all the residue class rings of powers of the ideal have FLC. We also give a combinatorial characterization of such ideals.
\end{abstract}

\section{INTRODUCTION}

Let $S=K\left[X_{1}, \ldots, X_{n}\right]$ be a polynomial ring over a field $K$ and $\mathfrak{m}=\left(X_{1}, \ldots, X_{n}\right)$. We view $(S, \mathfrak{m})$ as a standard graded algebra with the unique graded maximal ideal $\mathfrak{m}$. Let $I \subset S$ be a graded ideal and set $R=S / I$. Then $R$, or $I$, is called generalized Cohen-Macaulay or FLC if the local cohomology $H_{\mathfrak{m}}^{i}(R)$ has finite length for all $i<d=\operatorname{dim} R$. If $I$ is a Stanley-Reisner ideal, i.e., a square-free monomial ideal, it is well known that FLC coincides with Buchsbaumness, and Buchsbaum StanleyReisner ideals are well understood through topological characterization of the simplicial complexes corresponding to the ideal. However, FLC monomial ideals, which are not always square-free, have not been well understood. In [6], the second author gave combinatorial characterizations of FLC monomial ideals for $d \leq 3$ and a method for constructing FLC monomial ideals from Buchsbaum Stanley-Reisner ideals. But the problem of finding fairly large classes of FLC monomial ideals has been open.

The aim of this paper is to give a partial answer to this problem. As shown in 6], for a monomial ideal $I \subset S$, it is FLC only when $\sqrt{I}$ is a Buchsbaum Stanley-Reisner ideal. One of the typical cases of this situation is that $J \subset S$ is a Buchsbaum Stanley-Reisner ideal, and we consider its powers, i.e., $I=J^{\ell}$ for $\ell=1,2, \ldots$. Unfortunately, such a monomial ideal $J$ is not always FLC. We show that $I$ is FLC for all integers $\ell \geq 1$ if and only if the simplicial complex $\Delta$ corresponding to $J$ is pure and $k\left[\mathrm{k}_{\Delta}(\{i\})\right]$ is a complete intersection for all $i \in[n]=\{1, \ldots, n\}$ (Theorem [2.5). Then we classify such simplicial complexes $\Delta$ (Theorem [3.16).

For a Noetherian local ring $(R, \mathfrak{n})$ and a finitely generated $R$-module $M$, we denote by $\operatorname{Min}_{R} M$ the set of minimal primes in $\operatorname{Ass}_{R}(M)$. If $M$ is an Artinian $R$-module, we denote its length as an $R$-module by $\ell_{R}(M)$.

Received by the editors January 11, 2006 and, in revised form, April 13, 2006.

2000 Mathematics Subject Classification. Primary 13F55; Secondary 13 H10.

Key words and phrases. Stanley-Reisner ideal, monomial ideal, generalized Cohen-Macaulay ring, FLC ring, local cohomology.

(C)2007 American Mathematical Society 
We thank J. Herzog who read an earlier version of this manuscript and gave us valuable comments.

\section{Powers of a generically complete intersection ideal}

Let $I$ be an ideal of a Cohen-Macaulay local $\operatorname{ring}(S, \mathfrak{n})$ such that $I$ is generically a complete intersection, namely for arbitrary $\mathfrak{p} \in \operatorname{Min}_{S} S / I$, the ideal $I S_{\mathfrak{p}}$ is generated by an $S_{\mathfrak{p}}$-regular sequence. Here we consider the condition that when $S / I^{\ell}, \ell=$ $1,2, \ldots$, are Cohen-Macaulay. Now we recall the following well-known result, which was originally proved by Cowsik and Nori [3] and whose refinement as stated in the following lemma has been given, for example, in [1, 4, 8.

Lemma 2.1. Let $I \subset S$ be as above. Then the following conditions are equivalent:

(1) I is generated by an $S$-regular sequence,

(2) $S / I^{\ell+1}$ is Cohen-Macaulay for an arbitrary integer $\ell \geq 0$,

(3) $\Lambda=\left\{\ell \geq 0 \mid S / I^{\ell+1}\right.$ is Cohen-Macaulay $\}$ is an infinite set.

We will apply this result in the case that $S$ is the polynomial ring $k\left[X_{1}, \ldots, X_{n}\right]$ over a field $k$. By considering the standard grading, we can view $S$ as a positively graded $k$-algebra with the homogeneous maximal ideal $\mathfrak{n}=\left(X_{1}, \ldots, X_{n}\right)$. In this situation, we consider a graded ideal $I \subset S$. Recall that minimal primes of a graded ideal are graded. Then we obtain the definition of the graded version of a generically complete intersection in an obvious way.

Notice that the Stanley-Reisner ideal is a typical class of generically complete intersection (cf. Th. 5.1.4 [2]). Let $\Delta$ be a simplicial complex over the vertex set $[n]=\{1, \ldots, n\}$. We always assume that $\{i\} \in \Delta$ for all $i \in[n]$ unless otherwise stated. Then we denote the Stanley-Reisner ring corresponding to $\Delta$ by $k[\Delta]=$ $S / I_{\Delta}$, where $S=k\left[X_{1}, \ldots, X_{n}\right]$ is the polynomial ring over the field $k$ and $I_{\Delta} \subset S$ is the square-free monomial ideal corresponding to $\Delta$. For a monomial ideal $I \subset S$, we denote by $G(I)$ the minimal set of generators. Also for $F \in \Delta$, we define the link by $\operatorname{lk}_{\Delta}(F)=\{G \mid G \cup F \in \Delta, G \cap F=\emptyset\}$. See [2, Chapter 5, for the details on these terminologies.

Now we introduce the following notion.

Definition 2.2. A Stanley-Reisner ring $k[\Delta]$ is called a generalized complete intersection (gCI) if $\Delta$ is pure and $k\left[\mathrm{lk}_{\Delta}(\{i\})\right]$ is a complete intersection for all $i \in[n]$. We also call $\Delta$ a generalized complete intersection if $k[\Delta]$ is gCI.

This terminology comes from the analogy of generalized Cohen-Macaulayness. We notice the following fact, whose proof is left to the readers.

Proposition 2.3. For a simplicial complex $\Delta$, the following conditions are equivalent:

(i) $\Delta$ is $g C I$.

(ii) $\Delta$ is pure and $k[\Delta]_{P}$ is a complete intersection for every prime ideal $P(\neq$ $\mathfrak{m})$.

(iii) $\Delta$ is pure and $k[\Delta]_{X_{i}}$ is a complete intersection for every $i=1, \ldots, n$.

(iv) $\Delta$ is pure and if $G\left(I_{\Delta}\right)=\left\{u_{1}, \ldots, u_{\ell}\right\}$, then $\bar{u}_{1}, \ldots, \bar{u}_{\ell}$ is a $S_{i}$-regular sequence for all $i \in[n]$, where $S_{i}=k\left[X_{1}, \ldots, \stackrel{i}{\vee}, \ldots, X_{n}\right]$ and $\bar{u}_{j}$ is obtained from $u_{j}$ by substituting $X_{i}$ by 1 . 
Corollary 2.4. If $k[\Delta]$ is complete intersection, then it is a generalized complete intersection.

Now we can prove the main theorem of this section.

Theorem 2.5. Let $n \geq 1$ be an integer and let $\Delta$ be a simplicial complex over the vertex set $[n]$. Then the following conditions are equivalent:

(1) $k[\Delta]$ is a generalized complete intersection.

(2) $S / I_{\Delta}{ }^{\ell+1}$ has FLC for arbitrary integer $\ell \geq 0$.

(3) The set $\left\{\ell \geq 0 \mid\right.$ the ring $S / I_{\Delta}^{\ell+1}$ has FLC $\}$ is infinite.

If one of these conditions holds, $k[\Delta]$ is Buchsbaum.

Proof. $\quad(1) \Rightarrow(2)$ : Since $k[\Delta]_{x_{i}} \cong k\left[x_{i}, x_{i}^{-1}\right][1 \mathrm{k}(\{i\})]$, (1) is equivalent to the condition that $\Delta$ is pure and $k[\Delta]_{P}$ is a complete intersection for all prime ideals $P \neq \mathfrak{m}$. In particular, this implies that $k[\Delta]$ is Buchsbaum. See, for example, Excer. 5.3.15 2]. Also by Lemma 2.1, $S_{P} /\left(I_{\Delta} S_{P}\right)^{\ell}$ is CohenMacaulay for all integers $\ell \geq 0$. This implies that $S / I_{\Delta}^{\ell}$ is generalized Cohen-Macaulay, i.e., it has FLC.

$(3) \Rightarrow(1)$ : (3) implies that, for arbitrary $P \neq \mathfrak{m}, S_{P} /\left(I_{\Delta} S_{P}\right)^{\ell}$ is CohenMacaulay for infinitely may integers $\ell>0$. Thus by Lemma $2.1 k[\Delta]_{P}=$ $S_{P} / I_{\Delta} S_{P}$ must be a complete intersection, and this implies that $k[\Delta]_{x_{i}} \cong$ $k\left[x_{i}, x_{i}^{-1}\right]\left[\operatorname{lk}\left(\left\{x_{i}\right\}\right)\right]$ is a complete intersection for all $i=1, \ldots, n$. Then we obtain (1).

\section{Combinatorial Characterization}

The aim of this section is to give a combinatorial characterization of gCI simplicial complexes. We denote by $\Delta$ a simplicial complex over the vertex set $[n]$, and $S=k\left[X_{1}, \ldots, X_{n}\right]$ is the polynomial ring over the field $k$.

3.1. Core of a simplicial complex. We recall here the notion of a core of simplicial complexes (2] section 5.5). For $F \in \Delta$, we define $\operatorname{star}_{\Delta}(F)=\{G \mid G \cup F \in \Delta\}$. We also define core $[n]=\left\{i \in[n] \mid \operatorname{star}_{\Delta}(i) \neq \Delta\right\}$. Then the core of $\Delta$ is defined by core $\Delta=\{F \cap \operatorname{core}[n] \mid F \in \Delta\}$.

For two simplicial complexes $\Delta_{1}$ and $\Delta_{2}$ with disjoint vertex sets, we define the join of them by $\Delta_{1} * \Delta_{2}=\left\{F \cup G \mid F \in \Delta_{1}, G \in \Delta_{2}\right\}$. In particular, if $\Delta_{1}=\{\emptyset,\{i\}\}$, which we will abbreviate simply as $\Delta_{1}=\{i\},\{i\} * \Delta$ is called a cone over $\Delta$. Notice that if $i \in[n] \backslash \operatorname{core}[n]$, then we have

$$
\Delta=\operatorname{star}_{\Delta}(i)=\{G \mid G \cup\{i\} \in \Delta\}=\{i\} * \operatorname{link}_{\Delta}(i) .
$$

Thus if $\Delta \neq$ core $\Delta$, then $U:=[n] \backslash$ core $[n]$ is non-empty, and any element from $G(I)$ does not contain $X_{j}, j \in U$. This implies that $k[\Delta]$ is a polynomial ring over the ring $k[\operatorname{core} \Delta]: k[\Delta]=k[\operatorname{core} \Delta]\left[X_{j} \mid j \in U\right]$ with $k[\operatorname{core} \Delta]=S^{\prime} / I_{\Delta} S^{\prime}$, $S^{\prime}=k\left[X_{i} \mid i \in \operatorname{core}[n]\right]$.

3.2. Complete intersection by localization. Let $I_{\Delta}=\left(u_{1}, \ldots, u_{\ell}\right) \subset S$ be a square-free generalized complete intersection ideal that is not a complete intersection. Then, $(a) \Delta$ is pure, and $(b)$ the localization by $X_{i}, i \in[n]$, is a complete intersection. In this subsection, we consider the problem of characterizing $G\left(I_{\Delta}\right)$ with the ideal $I_{\Delta}$ having the property $(b)$. The purity condition $(a)$ will be considered in the next subsection. First of all we have 
Lemma 3.1. We must have $\ell \geq 2$ and $\Delta=\operatorname{core} \Delta$.

Proof. The necessity of the first condition is clear. Now assume that $\Delta \neq$ core $\Delta$. Then there exists an element $i \in[n] \backslash \bigcup_{j=1}^{\ell} \operatorname{supp}\left(u_{j}\right) . \quad k[\Delta]_{X_{i}}$ must be a complete intersection and $u_{1}, \ldots, u_{\ell}$ remains the minimal set of generators in $I_{\Delta} S_{X_{i}}$ so that $\operatorname{supp}\left(u_{j}\right), 1 \leq j \leq \ell$, must be pairwise disjoint. Thus we know that $k[\Delta]$ itself is a complete intersection, which contradicts the assumption.

Now we will assume $\Delta=$ core $\Delta$ in the following. We will rephrase our problem by a purely combinatorial setting. Let $S_{j}:=\operatorname{supp}\left(u_{j}\right), j=1, \ldots, \ell$, and set

$$
\mathcal{F}_{\Delta}:=\left\{S_{1}, \ldots, S_{\ell}\right\} \text {. }
$$

Since $\Delta=$ core $\Delta$, we have

$$
S_{1} \cup \cdots \cup S_{\ell}=[n]
$$

Also, since $\{i\} \in \Delta$ for all $i \in[n]$, we have

$$
\sharp S_{j} \geq 2 \quad \text { for all } 1 \leq j \leq \ell .
$$

Since $\left\{u_{1}, \ldots, u_{\ell}\right\}=G\left(I_{\Delta}\right)$, we have

$$
S_{i} \not \subset S_{j} \text { for all } i, j \text { with } i \neq j \text {. }
$$

Now for $i \in[n]$, we set $\mathcal{F}_{i}=\left\{S \backslash\{i\} \mid S \in \mathcal{F}_{\Delta}\right\}$. We will say that $S\left(\in \mathcal{F}_{\Delta}\right)$ is minimal in $\mathcal{F}_{i}$ if $(\emptyset \neq) S \backslash\{i\}$ is minimal in $\mathcal{F}_{i}$ with regard to the set inclusion. We set $\min \mathcal{F}_{i}:=\left\{S_{j} \backslash\{i\} \mid S_{j}\right.$ is minimal in $\left.\mathcal{F}_{i}\right\}$, which represents $G\left(I_{\Delta} S_{X_{i}}\right)$. Since $S_{X_{i}} /\left(u_{1}, \ldots, u_{\ell}\right) S_{X_{i}}$ is a complete intersection for all $i \in[n]$, we must have

$(S \backslash\{i\}) \cap\left(S^{\prime} \backslash\{i\}\right)=\emptyset \quad$ for all distinct $S \backslash\{i\}, S^{\prime} \backslash\{i\} \in \min \mathcal{F}_{i} \quad$ for all $i \in[n]$.

Finally, since we do not assume that $k[\Delta]$ itself is a complete intersection, we exclude the case that elements in $\mathcal{F}_{\Delta}$ are pairwise disjoint so that we pose

$$
S \cap S^{\prime} \neq \emptyset \quad \text { for some } S, S^{\prime} \in \mathcal{F}_{\Delta} \text {. }
$$

Now our problem is as follows: determine $\mathcal{F}_{\Delta}=\left\{S_{1}, \ldots, S_{\ell}\right\}, S_{j} \subset[n]$, satisfying the conditons (3.1), (3.2), (3.3), (3.4) and (3.5), together with some condition assuring the purity of $\Delta$.

Lemma 3.2. For every element $S \in \mathcal{F}_{\Delta}$, there exists another element $S^{\prime} \in \mathcal{F}_{\Delta}$ such that $S \cap S^{\prime} \neq \emptyset$.

Proof. Assume that $S_{1} \cap S_{j}=\emptyset$ for $j=2, \ldots, \ell$. Then for any $i \in S_{1}$ we have $i \notin S_{j}$ for $j=2, \ldots, \ell$. Thus $\mathcal{F}_{i}=\left\{S_{1} \backslash\{i\}, S_{2}, \ldots, S_{\ell}\right\}$ and, by the condition (3.3) and the assumption on $S_{1}$ we know that $\mathcal{F}_{i}=\min \mathcal{F}_{i}$. Thus, by the condition (3.4), we know that $S_{1}, \ldots, S_{\ell}$ are pairwise disjoint, which contradicts the condition (3.5).

Lemma 3.3. Let $S \in \mathcal{F}_{\Delta}$ be arbitrary. For any $i \in S, S$ is minimal in $\mathcal{F}_{i}$. Also if $S \backslash\{i\} \subset S^{\prime} \backslash\{i\}$ for distinct $S, S^{\prime} \in \mathcal{F}_{\Delta}$, we have $i \in S$.

Proof. Assume that $S$ with $i \in S$ is not minimal in $\mathcal{F}_{i}$. Then there exists another element $S^{\prime} \in \mathcal{F}_{\Delta}$ such that $S^{\prime} \backslash\{i\} \subset S \backslash\{i\}$. We must have $i \in S^{\prime}$ and $i \notin S$, since otherwise we have $S^{\prime} \subset S$ which contradicts (3.3). But this is impossible since we have $i \in S$ by assumption. Thus $S$ must be minimal in $\mathcal{F}_{i}$ with $i \in S$. The second assertion is clear from the above discussion. 
Lemma 3.4. For every $S, S^{\prime} \in \mathcal{F}_{\Delta}, \sharp\left(S \cap S^{\prime}\right) \leq 1$.

Proof. Assume that $i, j \in S_{1} \cap S_{2}, i \neq j$. Since $j \in\left(S_{1} \backslash\{i\}\right) \cap\left(S_{2} \backslash\{i\}\right) \neq \emptyset$, either $S_{1}$ or $S_{2}$ is non-minimal in $\mathcal{F}_{i}$ by the condition (3.4). But, since $i \in S_{1}$ and $i \in S_{2}$, they must be minimal in $\mathcal{F}_{i}$ by Lemma 3.3, a contradiction. Thus $S_{1} \cap S_{2}$ contains at most one element.

Lemma 3.5. Let $S \in \mathcal{F}_{\Delta}$ be such that $\sharp S \geq 3$. If $S^{\prime} \in \mathcal{F}_{\Delta}$ is another element such that $S^{\prime} \cap S \neq \emptyset$, then $\sharp S^{\prime}=2$.

Proof. Let $S_{1}, S_{2} \in \mathcal{F}_{\Delta}$ be such that $\sharp S_{1} \geq 3$ and $S_{1} \cap S_{2} \neq \emptyset$. Notice that the existence of such $S_{2}$ is assured by Lemma 3.2. Now by Lemma 3.4 we can assume without loss of generality that $S_{1} \cap S_{2}=\{1\}$ and $2,3 \in S_{1} \backslash S_{2}$. First we consider $\mathcal{F}_{2}$. Since $\left(S_{1} \backslash\{2\}\right) \cap\left(S_{2} \backslash\{2\}\right)=\{1\} \neq \emptyset$ and $2 \in S_{1}$, we must have $\left(S_{3} \backslash\{2\}\right) \subset\left(S_{2} \backslash\{2\}\right)$ for some $S_{3}\left(\neq S_{2}\right) \in \mathcal{F}_{\Delta}$ with $2 \in S_{3}$ by (3.4) and Lemma 3.3. We know that $S_{3} \neq S_{1}$. By Lemma 3.4 we know that $S_{3}=\{2, k\}$ with some $k \in S_{2} \backslash S_{1}$. We can assume without loss of generality that $k=4: S_{3}=\{2,4\}$. Next we consider $\mathcal{F}_{1}$. We have $\left(S_{1} \backslash\{1\}\right) \cap\left(S_{3} \backslash\{1\}\right)=\{2\} \neq \emptyset$ and $1 \in S_{1}$, so that by (3.4) and Lemma 3.3 we must have $\left(S_{4} \backslash\{1\}\right) \subset\left(S_{3} \backslash\{1\}\right)$ for some $S_{4}\left(\neq S_{3}\right) \in \mathcal{F}_{\Delta}$ with $1 \in S_{4}$. Thus by Lemma 3.4 we know that the only possible case is $S_{4}=S_{2}=\{1,4\}$, i.e., $\sharp S_{2}=2$ as required.

Lemma 3.6. Let $S \in \mathcal{F}_{\Delta}$ be such that $\sharp S \geq 3$. If $S^{\prime}=\{i, j\} \in \mathcal{F}_{\Delta}$ is another element such that $S^{\prime} \cap S=\{j\}$, then $\{i, k\} \in \mathcal{F}_{\Delta}$ for all $k \in S$.

Proof. As in the proof of Lemma 3.4 we can assume without loss of generality that $\sharp S_{1} \geq 3, S_{2}=\{1,2\}, 3,4 \in S_{1} \backslash S_{2}$ and $S_{1} \cap S_{2}=\{1\}$. It suffices to show that $\{2,3\} \in \mathcal{F}_{\Delta}$. We consider $\mathcal{F}_{3}$. Since $3 \in S_{1}, S_{1}$ is minimal in $\mathcal{F}_{3}$ by Lemma 3.3 . We have $\left(S_{1} \backslash\{3\}\right) \cap\left(S_{2} \backslash\{3\}\right)=\{1\} \neq \emptyset$ and $\left(S_{1} \backslash\{3\}\right) \not \subset\left(S_{2} \backslash\{3\}\right)$. Thus, by (3.4) and Lemma 3.3, we have $\left(S_{3} \backslash\{3\}\right) \subset\left(S_{2} \backslash\{3\}\right)=S_{2}$ for some $S_{3} \in \mathcal{F}_{\Delta}$ with $3 \in S_{3}$. By Lemma 3.4, we know that $S_{3}=\{2,3\}$.

Now for an element $S \in \mathcal{F}_{\Delta}$ such that $\sharp S \geq 3$, we set

$$
\mathcal{C}(S)=\left\{i \in[n] \mid\{i, j\} \in \mathcal{F}_{\Delta} \text { for some } j \in S\right\} .
$$

By the condition (3.3), we know $\mathcal{C}(S) \cap S=\emptyset$. According to Lemma 3.6. for any $i \in \mathcal{C}(S)$, we have $\{i, k\} \in \mathcal{F}_{\Delta}$ for all $k \in S$.

Lemma 3.7. Let $S \in \mathcal{F}_{\Delta}$ be such that $\sharp S \geq 3$. For an $i \in[n]$, if $i \notin \mathcal{C}(S) \cup S$, then $\{i, k\} \in \mathcal{F}_{\Delta}$ for all $k \in \mathcal{C}(S)$.

Proof. Assume that for an $i \notin \mathcal{C}(S)$ there exists $k \in \mathcal{C}(S)$ such that $\{i, k\} \notin \mathcal{F}_{\Delta}$. We will deduce a contradiction. By (3.1) there exists at least one $T \in \mathcal{F}_{\Delta}$ such that $i \in T$. Now we have two cases: $(i)\{i, k\} \subset T$ and $\sharp T \geq 3$, or (ii) for all such $T$ we have $\{i, k\} \not \subset T$.

We first consider the case $(i)$. We have $\{k, j\} \in \mathcal{F}_{\Delta}$ for some $j \in S$, and $k \in T$ with $\sharp T \geq 3$. Thus by Lemma $3.6\{i, j\} \in \mathcal{F}_{\Delta}$, which contradicts the assumption that $i \notin \mathcal{C}(S)$.

Next we consider the case (ii). For any distinct $j_{1}, j_{2} \in S$, we have $\left(\left\{k, j_{1}\right\} \backslash\{i\}\right) \cap$ $\left(\left\{k, j_{2}\right\} \backslash\{i\}\right)=\{k\} \neq \emptyset$ in $\mathcal{F}_{i}$. Thus there exists $S^{\prime} \in \mathcal{F}_{\Delta}$ with $i \in S^{\prime}$ such that $\left(S^{\prime} \backslash\{i\}\right) \subset\left(\left\{k, j_{p}\right\} \backslash\{i\}\right)$ with $p=1$ or 2 . Thus we must have either $S^{\prime}=\{i, k\}$ or $S^{\prime}=\left\{i, j_{p}\right\}$. But both of the cases contradict the assumptions of non-existence of $\{i, k\} \in \mathcal{F}_{\Delta}$ and $i \notin \mathcal{C}(S)$. 
In the following, we partly use the language of graph theory. We call an element $E \in \mathcal{F}_{\Delta}$ with $\sharp E=2$ an edge. Also we will call a set $P=\left\{E_{1}, \ldots, E_{q}\right\}$ of edges with $E_{i} \cap E_{i+1} \neq \emptyset, i=1, \ldots, q-1$, a path.

Lemma 3.8. Any two elements $i, j \in[n]$ are linked with a path $P=\left\{E_{1}, \ldots, E_{q}\right\}$, such that $i \in E_{1}$ and $j \in E_{q}$.

Proof. The case that $\{i, j\} \in \mathcal{F}_{\Delta}$ is trivial. Also, if $\{i, j\} \subset S$ for some $S \in \mathcal{F}_{\Delta}$ with $\sharp S \geq 3$, then by Lemmas 3.2, 3.5 and 3.6, there exists $k \in \mathcal{C}(S)$ and we have $\{i, k\},\{k, j\} \in \mathcal{F}_{\Delta}$. Namely, $i$ and $j$ are linked with the path $P=\{\{i, k\},\{k, j\}\}$. Now we will consider other cases.

Let $i, j \in[n]$ be arbitrary. Assume that there exists a set $Q=\left\{S_{1}, \ldots, S_{r}\right\}$, $r \geq 2$, of elements from $\mathcal{F}_{\Delta}$ such that $i \in S_{1}, j \in S_{r}$ and $S_{i} \cap S_{i+1} \neq \emptyset$ for $i=1, \ldots, r-1$, where we have $\sharp S_{j} \geq 3$ for some $1 \leq j \leq r$. By Lemma 3.5, we must have $\sharp S_{j-1}=\sharp S_{j+1}=2$ if $S_{j-1}$ and $S_{j+1}$ exist. We consider the case that $S_{j-1}$ exists. One proves the other case in a similar way. We set $S_{j-1}=\left\{i_{1}, i_{2}\right\}$ and $S_{j}=\left\{i_{2}, \ldots, i_{q}\right\}$ with $q \geq 4$. Then by Lemma 3.6 there exists $\left\{i_{1}, i_{q}\right\} \in \mathcal{F}_{\Delta}$. Thus by replacing the pair of two sets $S_{j-1}, S_{j}$ by an edge $\left\{i_{1}, i_{q}\right\}$ we can remove $S_{j}$ from $Q$. Using the same argument, we can construct from $Q$ a path $P$ with the required property.

Finally we show the existence of the path. Assume that if there exist $i, j \in$ $[n]$ that are not linked by any path, i.e., contained in different components, then considering $\mathcal{F}_{i}$ or $\mathcal{F}_{j}$ we immediately know that elements from $\mathcal{F}_{\Delta}$ contained in each component must be pairwise disjoint, which entails that all the elements of $\mathcal{F}_{\Delta}$ are pairwise disjoint. But this contradicts the condition (3.5).

Lemma 3.9. If there exists a length 4 path $P=\left\{\left\{i_{p}, i_{p+1}\right\} \in \mathcal{F}_{\Delta} \mid p=1,2,3,4\right\}$, then there exists an edge $\left\{i_{1}, i_{q}\right\} \in \mathcal{F}_{\Delta}$ with $q=3,4$ or 5 .

Proof. Consider $\mathcal{F}_{i_{1}}$. We have $\left(\left\{i_{3}, i_{4}\right\} \backslash\left\{i_{1}\right\}\right) \cap\left(\left\{i_{4}, i_{5}\right\} \backslash\left\{i_{1}\right\}\right)=\left\{i_{4}\right\} \neq \emptyset$. Thus by Lemma 3.3 and the condition (3.4) there exists $S^{\prime} \in \mathcal{F}_{\Delta}$ with $i_{1} \in S^{\prime}$ such that $\left(S^{\prime} \backslash\left\{i_{1}\right\}\right) \subset\left(\left\{i_{3}, i_{4}\right\} \backslash\left\{i_{1}\right\}\right)=\left\{i_{3}, i_{4}\right\}$ or $\left(S^{\prime} \backslash\left\{i_{1}\right\}\right) \subset\left(\left\{i_{4}, i_{5}\right\} \backslash\left\{i_{1}\right\}\right)=\left\{i_{4}, i_{5}\right\}$. Thus, by taking the condition (3.3) into account, we know that $S^{\prime}=\left\{i_{1}, i_{q}\right\}$ with $q=3,4$ or 5 .

Now we show a converse to what we have proved:

Proposition 3.10. Consider $\mathcal{F}_{\Delta}=\left\{S_{j} \mid S_{j} \subset[n], j=1, \ldots, \ell\right\}$ satisfying the following conditions, which are the same as (3.1), (3.2) and (3.3):

1. $S_{1} \cup \cdots \cup S_{\ell}=[n]$,

2. $\sharp S_{j} \geq 2$ for all $1 \leq j \leq \ell$, and

3. $S_{i} \not \subset S_{j}$ for all $i, j$ with $i \neq j$.

Then the following conditions are equivalent:

(i) $\mathcal{F}_{\Delta}$ satisfies the following conditions, which are the same as (3.4) and (3.5):

(a) $(S \backslash\{i\}) \cap\left(S^{\prime} \backslash\{i\}\right)=\emptyset$ for all distinct $S \backslash\{i\}$ and $S^{\prime} \backslash\{i\} \in \min \mathcal{F}_{i}$ for all $i \in[n]$,

(b) $S \cap S^{\prime} \neq \emptyset$ for some $S, S^{\prime} \in \mathcal{F}_{\Delta}$.

(ii) For $\mathcal{F}_{\Delta}$, Lemma 3.2 to Lemma 3.9 hold.

Proof. We have only to show (i) from (ii). The condition (3.5) is immediate from Lemma 3.2. Now we show the condition (3.4) from Lemmas 3.2 3.9 . 
Assume that there exists $i \in[n]$ and $S, T \in \mathcal{F}_{\Delta}$ such that

$$
(S \backslash\{i\}) \cap(T \backslash\{i\}) \neq \emptyset
$$

and both $S$ and $T$ are minimal in $\mathcal{F}_{i}$. If $i \in S \cap T$, then (3.6) implies $\sharp(S \cap T) \geq 2$, which contradicts Lemma 3.4. Thus we have either $i \notin S$ or $i \notin T$, and we can refine (3.6) as

$$
(S \backslash\{i\}) \cap(T \backslash\{i\})=S \cap T=\{j\}
$$

for some $j$. Now we have two cases:

Case (either $\sharp S \geq 3$ or $\sharp T \geq 3$ ): We can assume that $\sharp S \geq 3$. Then $\sharp T=2$ by Lemma 3.5 so that we set $T=\{j, k\}$ for some $k \in[n]$. If $k=i$, then $i \notin S$ since otherwise we have $T \subset S$, contradinting the condition (3.3). Then we have $\{j\}=(T \backslash\{i\}) \subset(S \backslash\{i\})=S$, which contradicts the assumption that $S$ is minimal in $\mathcal{F}_{i}$. Thus we must have $k \neq i$.

Now assume that $i \in S$. Then by Lemma 3.6 we have $\{i, k\} \in \mathcal{F}_{\Delta}$, so that $\{k\}=\{i, k\} \backslash\{i\} \subset T \backslash\{i\}=\{k, j\}$ and $\{i, k\} \neq T$, which contradicts the minimality of $T$ in $\mathcal{F}_{i}$. Thus we must have $i \notin S$. Moreover, if $i \in \mathcal{C}(S)$, then again by Lemma 3.6 we have $\{i, j\} \in \mathcal{F}_{\Delta}$ and $\{j\}=\{i, j\} \backslash\{i\} \subset$ $T \backslash\{i\}=\{k, j\}$ and $\{i, j\} \neq T$, which contradicts the minimality of $T$ in $\mathcal{F}_{i}$. Thus we must have $i \notin \mathcal{C}(S) \cup S$. Then by Lemma 3.7 we have $\{k, i\} \in \mathcal{F}_{\Delta}$, so that $\{k\}=\{k, i\} \backslash\{i\} \subset T \backslash\{i\}=\{k, j\}$ and $\{k, i\} \neq T$. This contradicts the minimality of $T$ in $\mathcal{F}_{i}$.

Case $(\sharp S=\sharp T=2)$ : We set $S=\{j, h\}$ and $T=\{j, k\}$ for some $h, k(\neq i) \in$ $[n]$. Then by Lemma $3.8 i$ and $h$ are linked with a path $P=\left\{E_{1}, \ldots, E_{r}\right\}$, with edges $E_{j} \in \mathcal{F}_{\Delta}, j=1, \ldots, r, E_{k} \cap E_{k+1} \neq \emptyset$ for $k=1, \ldots, r-1$ such that $i \in E_{1}$ and $h \in E_{r}$. If $r \geq 4$, we can take another path with smaller length by Lemma 3.9, so that we can assume that $r \leq 3$. Then $P \cup\{S, T\}$ is a path of length $\leq 5$ that links $i$ and $k$. Again by using Lemma 3.9 we know that $\{i, l\} \in \mathcal{F}_{\Delta}$ where $l=h, j$ or $k$. But then we have $\{i, l\} \backslash\{i\} \subset S$ or $\{i, l\} \backslash\{i\} \subset T$, and $S$ or $T$ is not minimal in $\mathcal{F}_{i}$, which contradicts the assumption.

3.3. Purity of $\Delta$. We now characterize the purity of the simplicial complex. For $\mathcal{F}_{\Delta}$ as in Proposition 3.10, we define the associated graph $G_{\Delta}$ as follows: The vertex set is $V\left(G_{\Delta}\right)=[n]$ and the edge set is $E\left(G_{\Delta}\right)=\left\{\{i, j\} \mid\{i, j\} \notin \mathcal{F}_{\Delta}\right\}$. Then $G_{\Delta}$ is exactly the 1-skeleton of $\Delta: G_{\Delta}=\{F \in \Delta \mid \operatorname{dim} F \leq 1\}$.

Notice in particular that, for $S \in \mathcal{F}_{\Delta}$ with $\sharp S \geq 3$, all the edges in the complete graph $\mathcal{K}(S)$ over the vertex set $S$ are contained in $E\left(G_{\Delta}\right)$.

Remark 3.11. Recall that if $I_{\Delta}$ is generated in degree 2, it is called an edge ideal represented by a graph $G$ whose edges are supports of the generators. In this case, $G_{\Delta}$ is nothing but the complement $\bar{G}$ (see [7] Chapter 6).

Definition 3.12. For a finite graph $G$ we define

$$
\operatorname{Simp}(G)=\left\{\begin{array}{l}
F \mid \begin{array}{l}
\exists H \text { a subgraph of } G \text { such that } \\
F=V(H) \text { and } H \sim \mathcal{K}_{r} \text { for some } r
\end{array}
\end{array}\right\}
$$

where $\mathcal{K}_{r}$ is the complete graph over the vertex set $[r]$ and $G \sim H$ denotes an isomorphism of graphs. 
Definition 3.13. For $\mathcal{F}_{\Delta}$ as in Proposition 3.10 and a simplicial complex $\Gamma$ over the vertex set $[n]$, we define

$$
\operatorname{Red}\left(\mathcal{F}_{\Delta}, \Gamma\right)=\Gamma \backslash\left\{F \in \Gamma \mid S \subset F \text { for some } S \in \mathcal{F}_{\Delta} \text { with } \sharp S \geq 3\right\} \text {. }
$$

Proposition 3.14 (cf. Prop. 6.1.25 [7]). For $\mathcal{F}_{\Delta}$ as in Proposition 3.10 , we have

$$
\Delta=\operatorname{Red}\left(\mathcal{F}_{\Delta}, \operatorname{Simp}\left(G_{\Delta}\right)\right) .
$$

Proof. $\Delta \subset \operatorname{Red}\left(\mathcal{F}_{\Delta}, \operatorname{Simp}\left(G_{\Delta}\right)\right)$ is clear from the definitions. Now take any $F=\left\{i_{1}, \ldots, i_{r}\right\} \in \operatorname{Red}\left(\mathcal{F}_{\Delta}, \operatorname{Simp}\left(G_{\Delta}\right)\right)$. Since $F \in \operatorname{Simp}\left(G_{\Delta}\right),\left\{i_{p}, i_{q}\right\} \in \Delta$, i.e., $\left\{i_{p}, i_{q}\right\} \notin \mathcal{F}_{\Delta}$, for all $1 \leq p<q \leq r$. In other words, $X_{i_{p}} X_{i_{q}} \notin I_{\Delta}$ for all $1 \leq p<q \leq r$. If $F \notin \Delta$, then the monomial $X_{i_{1}} \cdots X_{i_{r}}$ is in $I_{\Delta}$. Thus there exists a subset $S=\left\{j_{1}, \ldots, j_{s}\right\} \subset\left\{i_{1}, \ldots, i_{r}\right\}$ with $3 \leq s \leq r$ such that $S \in \mathcal{F}_{\Delta}$. But this contradicts the assumption that $F \in \operatorname{Red}\left(\mathcal{F}_{\Delta}, \operatorname{Simp}\left(G_{\Delta}\right)\right)$. Thus $F \in \Delta$ as required.

Corollary 3.15. For $\mathcal{F}_{\Delta}$ as in Propostion 3.10 , the following conditions are equivalent:

(i) $\Delta$ is pure,

(ii) for every vertex $i \in G_{\Delta}$ consider a subgraph $H_{i} \subset G_{\Delta}$ such that

(a) $H_{i} \cong \mathcal{K}_{r_{i}}$ and $i \in V\left(H_{i}\right)$,

(b) $V\left(H_{i}\right)$ contains no subset $S \in \mathcal{F}_{\Delta}$ such that $\sharp S \geq 3$, and

(c) $r_{i}$ is maximal among other such subgraphs.

Then $r_{i}$ is constant for all $i$.

Proof. By Proposition 3.14 we know that a subgraph $H\left(\subset G_{\Delta}\right)$ isomorphic to $\mathcal{K}_{r}$ satisfying the conditions given above is exactly the skeleton of an $(r-1)$-face in $\Delta$.

3.4. Combinatorial characterization of a generalized complete intersection. Now we obtain a combinatorial characterization of a generalized complete intersection Stanley-Reisner ring.

Theorem 3.16. Let $k[\Delta]$ be a Stanley-Reisner ring with $\Delta=$ core $\Delta$. Let $G\left(I_{\Delta}\right)=$ $\left\{u_{1}, \ldots, u_{\ell}\right\}$ and $\mathcal{F}_{\Delta}=\left\{\operatorname{supp}\left(u_{j}\right) \mid j=1, \ldots, \ell\right\}$. Assume that $k[\Delta]$ is not a complete intersection. Then $k[\Delta]$ is a generalized complete intersection if and only if the following conditions hold:

1. For every $S \in \mathcal{F}_{\Delta}$ with $\sharp S \geq 3$, there exists a non-empty $\operatorname{set} \mathcal{C}(S)(\subset[n])$ such that

(a) $\mathcal{C}(S) \cap S=\emptyset$,

(b) for every $i \in \mathcal{C}(S)$, we have $E_{i j}:=\{i, j\} \in \mathcal{F}_{\Delta}$ for all $j \in S$. Moreover if $S \cap T \neq \emptyset$ for $T \in \mathcal{F}_{\Delta}$, then $T=E_{i j}$ for some $i, j$,

(c) for every $k \notin \mathcal{C}(S) \cup S$, we have $\{i, k\} \in \mathcal{F}_{\Delta}$ for all $i \in \mathcal{C}(S)$.

2. Any two elements $i, j \in[n]$ are linked with a path $P=\left\{\left\{i_{k}, i_{k+1}\right\} \mid k=\right.$ $1, \ldots, r\}$, with edges $\left\{i_{k}, i_{k+1}\right\} \in \mathcal{F}_{\Delta}$ for $k=1, \ldots, r$ such that $i=i_{1}$ and $j=i_{r+1}$.

3. If there exists a length 4 path $P=\left\{\left\{i_{p}, i_{p+1}\right\} \in \mathcal{F}_{\Delta} \mid p=1,2,3,4\right\}$, then there must be an edge $\left\{i_{1}, i_{q}\right\} \in \mathcal{F}_{\Delta}$ with $q=3,4$ or 5 .

4. For every vertex $i \in G_{\Delta}$ consider a subgraph $H_{i} \subset G_{\Delta}$ such that

(a) $H_{i} \cong \mathcal{K}_{r_{i}}$ and $i \in V\left(H_{i}\right)$,

(b) $V\left(H_{i}\right)$ contains no subset $S \in \mathcal{F}_{\Delta}$ such that $\sharp S \geq 3$, and

(c) $r_{i}$ is maximal among other such subgraphs. 
Then $r_{i}$ is constant for all $i$.

Moreover, if $\Delta \neq$ core $\Delta, k[\Delta]$ is a generalized complete intersection if and only if it is a complete intersection.

Proof. The main part is straightforward by Propositions 3.10 and 3.14. The last part is by Corollary 2.4 and Lemma 3.1 .

We show a few examples of generalized complete intersection Stanley-Reisner ideals.

Example 3.17. Examples of non-Cohen-Macaulay edge ideals:

1. $I_{\Delta}=\left(X_{1} X_{3}, X_{1} X_{4}, X_{2} X_{3}, X_{2} X_{4}\right)=\left(X_{1}, X_{2}\right) \cap\left(X_{3}, X_{4}\right) \subset k\left[X_{1}, \ldots, X_{4}\right]$ and $\Delta=\langle\{1,2\},\{3,4\}\rangle$ (two disjoint edges).

2. $I_{\Delta}=\left(X_{1} X_{2}, X_{2} X_{3}, X_{1} X_{3}, X_{3} X_{4}, X_{4} X_{5}, X_{1} X_{5}\right)=\left(X_{2}, X_{3}, X_{5}\right) \cap\left(X_{1}, X_{3}\right.$, $\left.X_{5}\right) \cap\left(X_{1}, X_{3}, X_{4}\right) \cap\left(X_{1}, X_{2}, X_{4}\right) \subset k\left[X_{1}, \ldots, X_{5}\right]$ and $\Delta=\langle\{1,4\},\{4,2\}$, $\{2,5\},\{5,3\}\rangle$ (a path of length 4 ).

3. $I_{\Delta}=\left(X_{1} X_{2}, X_{2} X_{3}, X_{1} X_{3}, X_{3} X_{4}, X_{4} X_{5}, X_{1} X_{5}, X_{2} X_{5}\right)=\left(X_{2}, X_{3}, X_{5}\right) \cap$ $\left(X_{1}, X_{3}, X_{5}\right) \cap\left(X_{1}, X_{2}, X_{4}\right) \subset k\left[X_{1}, \ldots, X_{5}\right]$ and $\Delta=\langle\{1,4\},\{4,2\},\{5,3\}\rangle$ (a disjoint union of a path of length 2 and an edge).

4. $I_{\Delta}=\left(X_{1} X_{2}, X_{1} X_{5}, X_{2} X_{3}, X_{2} X_{5}, X_{3} X_{4}\right)=\left(X_{2}, X_{4}, X_{5}\right) \cap\left(X_{2}, X_{3}, X_{5}\right) \cap$ $\left(X_{1}, X_{2}, X_{4}\right) \cap\left(X_{1}, X_{2}, X_{3}\right) \cap\left(X_{1}, X_{3}, X_{5}\right) \subset k\left[X_{1}, \ldots, X_{5}\right]$ and $\Delta=\langle\{1,3\},\{3,5\},\{5,4\},\{4,1\}\{4,2\}\rangle$ (an edge attached to a circle).

5. $I_{\Delta}=\left(X_{1}, \ldots, X_{n}\right) \cap\left(X_{n+1}, \ldots, X_{2 n}\right) \subset k\left[X_{1}, \ldots, X_{2 n}\right]$. Notice that $G\left(I_{\Delta}\right)$ is a bipartite graph.

Example 3.18. Examples of Cohen-Macaulay edge ideals:

1. $I_{\Delta}=\left(X_{1} X_{2}, X_{2} X_{3}, X_{3} X_{4}\right)=\left(X_{2}, X_{4}\right) \cap\left(X_{2}, X_{3}\right) \cap\left(X_{1}, X_{3}\right) \subset k\left[X_{1}, \ldots\right.$, $\left.X_{4}\right]$ and $\Delta=\langle\{1,3\},\{1,4\},\{4,2\}\rangle$ (a path of length 3).

2. $I_{\Delta}=\left(X_{1} X_{2}, X_{2} X_{3}, X_{3} X_{4}, X_{4} X_{5}, X_{5} X_{1}\right)=\left(X_{2}, X_{4}, X_{5}\right) \cap\left(X_{1}, X_{2}, X_{4}\right) \cap$ $\left(X_{1}, X_{3}, X_{4}\right) \cap\left(X_{1}, X_{3}, X_{5}\right) \cap\left(X_{2}, X_{3}, X_{5}\right) \subset k\left[X_{1}, \ldots, X_{5}\right]$ and $\Delta=\langle\{1,3\},\{3,5\},\{5,2\},\{2,4\},\{4,1\}\rangle$ (a circle).

For Cohen-Macaulay edge ideals, see [7].

Example 3.19. Ideals whose generators contain degree $\geq 3$ monomials:

1. $I_{\Delta}=\left(X_{1} X_{2} X_{3}\right)+\left(X_{1}, X_{2}, X_{3}\right) *\left(X_{4}, X_{5}, X_{6}\right)+\left(X_{4} X_{7}, X_{5} X_{7}, X_{6} X_{7}\right) \subset$ $k\left[X_{1}, \ldots, X_{7}\right]$ and

$$
\Delta=\langle\{1,2,7\},\{1,3,7\},\{2,3,7\},\{4,5,6\}\rangle,
$$

which is a not Cohen-Macaulay complex since it is not connected.

2. $I_{\Delta}=\left(X_{1} X_{2} X_{3} X_{4}\right)+\left(X_{1}, X_{2}, X_{3}, X_{4}\right) *\left(X_{5}, X_{6}, X_{7}\right)$ and

$$
\Delta=\langle\{1,2,3\},\{1,2,4\},\{1,3,4\},\{2,3,4\},\{5,6,7\}\rangle,
$$

which is a not Cohen-Macaulay complex since it is not connected.

\section{REFERENCES}

1. R. Achilles and W. Vogel, Über vollständige Durchschnitte in lokalen Ringen. Math. Nachr. 89 (1979), 285-298. MR0546888 (82e:14060)

2. W. Bruns and J. Herzog, Cohen-Macaulay Rings, Revised version, Cambridge Studies in Advanced Mathematics, 39. Cambridge University Press, Cambridge, 1998. MR.1251956 (95h:13020)

3. R. C. Cowsik and M. V. Nori, On the fibres of blowing up. J. Indian Math. Soc. (N.S.) 40 (1976), no. 1-4, 217-222 (1977). MR0572990(58:28011) 
4. M. Herrmann and U. Orbanz, Faserdimensionen von Aufblasungen lokaler Ringe und Äquimultiplizitat. J. Math. Kyoto Univ. 20 (1980), no. 4, 651-659. MR0592352 (83k:13016)

5. R. P. Stanley, Combinatorics and commutative algebra, Second edition. Progress in Mathematics, 41. Birkhäuser Boston, Inc., Boston, MA, 1996. MR.1453579 (98h:05001)

6. Y. Takayama, Combinatorial characterizations of generalized Cohen-Macaulay monomial ideals, Bull. Math. Soc. Sci. Math. Roumanie (N.S.) 48(96) (2005), no. 3, 327-344. MR2165349 (2006e:13017)

7. R. H. Villarreal, Monomial Algebras, Monographs and Textbooks in Pure and Applied Mathematics, 238. Marcel Dekker, Inc., New York, 2001. MR.1800904(2002c:13001)

8. R. Waldi, Vollständige Durchschnitte in Cohen-Macaulay-Ringen. Arch. Math. (Basel) 31 (1978/79), no. 5, 439-442. MR0526608 (80d:14029)

Department of Mathematics, School of Science and Technology, Meiji University, 214-8571, JAPAN

E-mail address: goto@math.meiji.ac.jp

Department of Mathematical Sciences, Ritsumeikan University, 1-1-1 Nojihigashi, Kusatsu, Shiga 525-8577, JAPAN

E-mail address: takayama@se.ritsumei.ac.jp 